\title{
EDS Analysis at Low Beam Currents Facilitated by a Large Area Silicon Drift Detector
}

\author{
Jon J. McCarthy ${ }^{1}$ and John F. Konopka ${ }^{2}$ \\ 1. University of Wisconsin, Madison WI. \\ 2. Thermo Fisher Scientific, Madison WI.
}

EDS x-ray analysis suffers from the relative rarity of x-rays emitted from a sample during an analysis. A large number of electrons impact with a sample for each X-ray that is captured and measured by the EDS detector. For many samples the solution to obtaining more counts is simply to increase the beam current as $\mathrm{x}$-ray production is directly related to the number of electrons striking the sample. Modern SEMS can easily produce tens of nanoamps of beam current. However, in many cases high beam currents can damage the sample. Biological samples are easily damaged by the electron beam. In many glassy samples, $\mathrm{Na}$ and other elements becomes mobile under the influence of large beam currents. Albite and many other minerals suffer alteration when exposed to high beam currents. The resolution of tungsten gun SEMs suffers at larger beam currents. Instead of raising the beam current an alternate solution is to use a larger detector. This works well up to a point. As the diameter of a round detector increases it must be moved farther back from a sample in order to avoid collision with the SEM pole piece. One solution is to change the shape of the detector. A rectangular detector can be located nearer the sample, since the wedge shape of the tube allows it be inserted closer under the pole piece. The combination of a large area and a short sample to detector distance yields a very large solid angle and this allows capturing more x-rays with less beam current.

A property of the Silicon Drift Detector (SDD) technology is that such shapes are possible while maintaining excellent resolution. A detector of rectangular shape was tested on a sample of cover glass for cell phones. The BSE image is shown in Figure 1 and the net counts $\mathrm{x}$-ray maps are shown in Figure 2. This data was taken at $10 \mathrm{kV}$ and $160 \mathrm{pA}$ with a Thermo Scientific NSS EDS analyzer and a $100 \mathrm{~mm}^{2}$ UltraDry "racetrack" SDD mounted on a Leo 1455 VP SEM. The total count rate was about 18 kcps. With two of these detectors mounted on one SEM, good x-ray maps would be obtained at less than $100 \mathrm{pA}$ beam current. This allows analysis of beam sensitive samples in a reasonable time [1].

The data characterize this field of view. Sufficient data is present in the Spectral Image to calculate net counts for these elements by removing the x-ray continuum background and separating overlapped peaks. A line profile for $\mathrm{K}$ extracted from the map data shows the diffusion depth of $\mathrm{K}$ into the glass surface. With a standard detector, a beam current of about 5 to $10 \mathrm{nA}$ would be required to collect this quality of data $[2]$ !

References:

[1] S. M. Seddio, P. K. Carpenter, Microsc. Microanal. 23 (2017), p. 1012.

[2] The authors acknowledge Thermo Fisher Scientific for use of their Madison Demo Lab. 


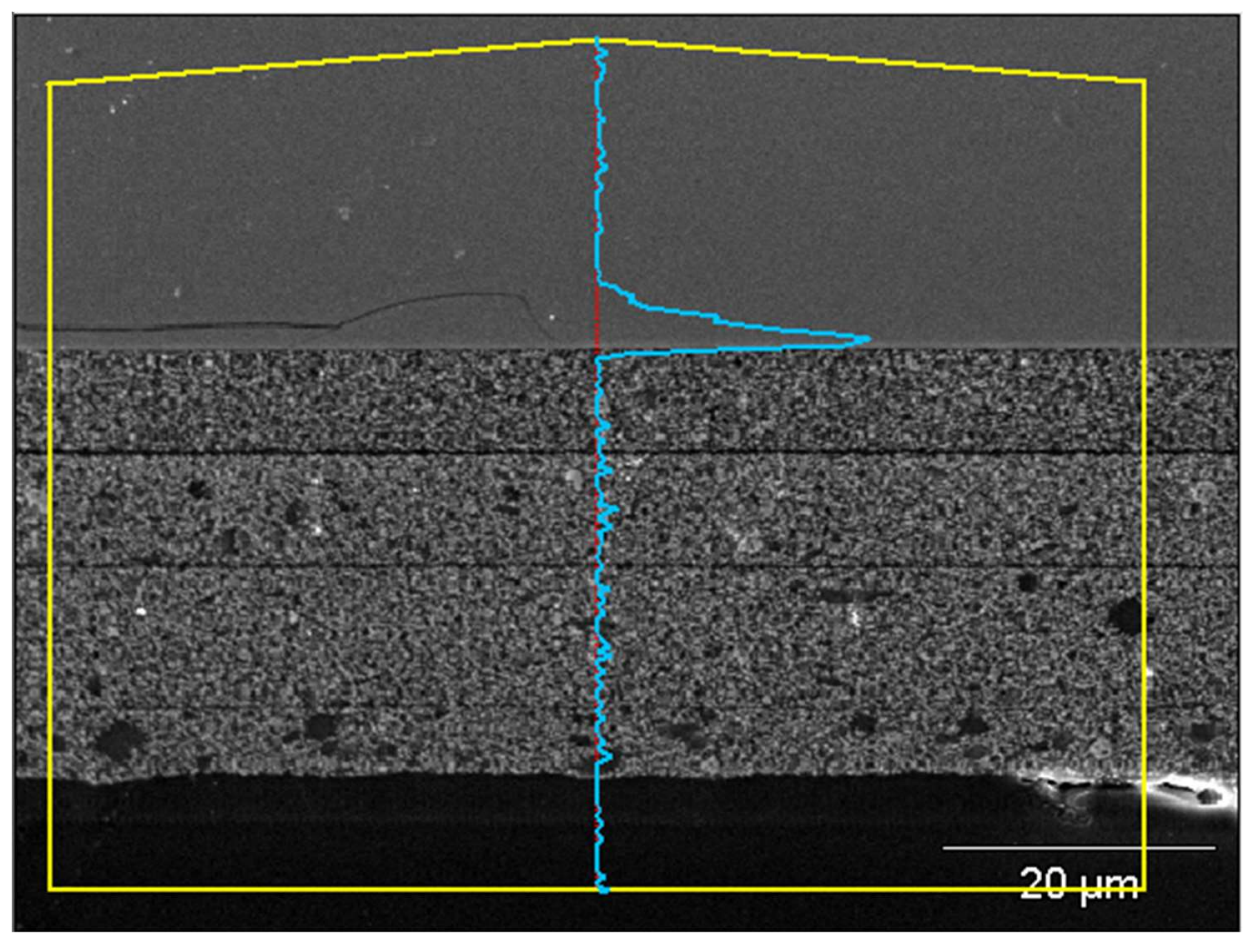

Figure 1. Overlay of $\mathrm{K}$ line profile extracted from the $\mathrm{x}$-ray map overlaid on the BSE image of the surface of a cross section of a cell phone glass protector. The glass body is at the top.

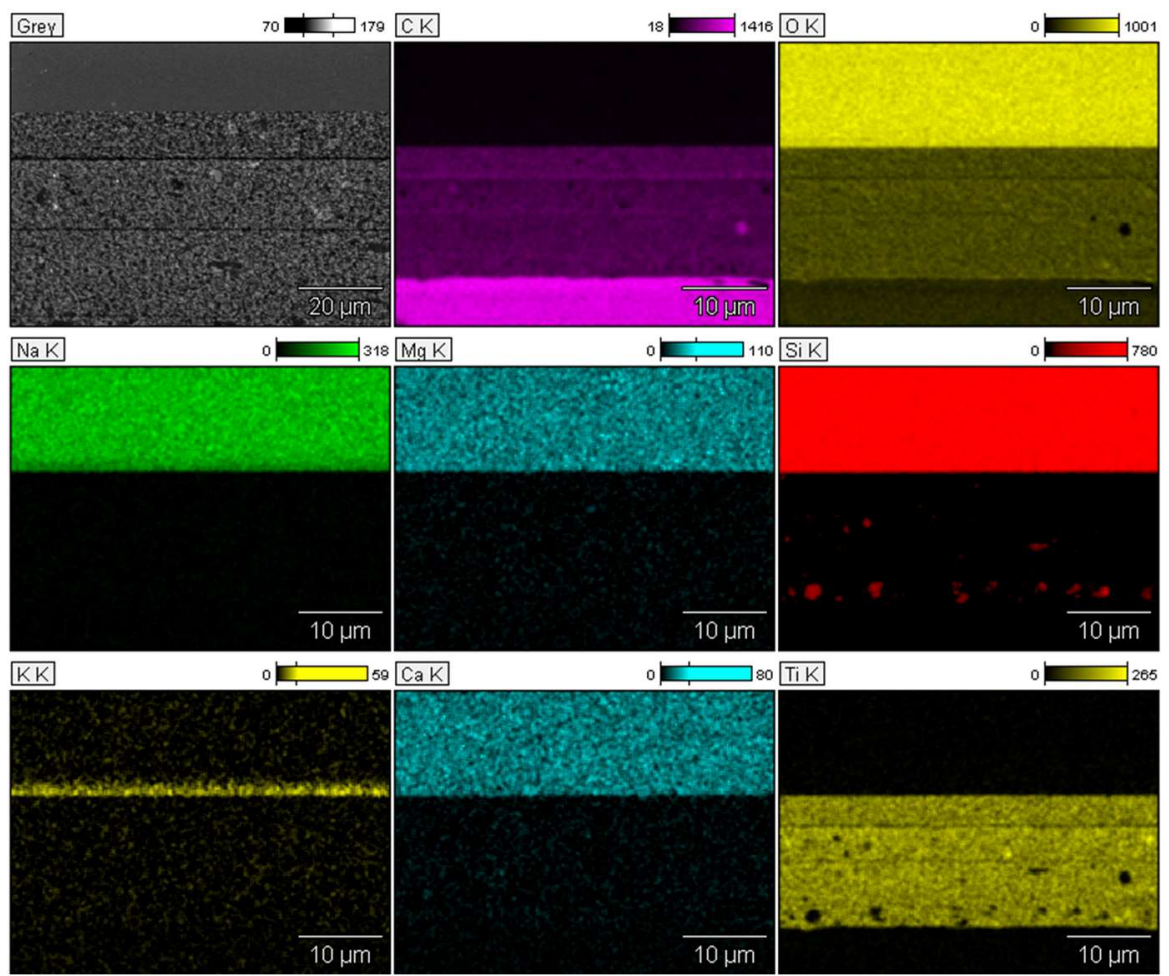

Figure 2. Net counts elemental maps of the image shown in Figure 1. Data was acquired at 10 $\mathrm{kV}$ with a beam current of $160 \mathrm{pA}$. Map resolution 256 x 192. Map time about 10 minutes. 